\title{
VLBI Frequency Transfer using CONT11
}

\author{
Carsten Rieck $^{1,2}$, Rüdiger Haas ${ }^{2}$, Per Jarlemark ${ }^{1}$ and Kenneth Jaldehag ${ }^{1}$ \\ ${ }^{1}$ Measurement Technology \\ SP Technical Research Institute of Sweden \\ Box 857, SE-50115 Borås, Sweden \\ ${ }^{2}$ Chalmers University of Technology \\ Department of Earth and Space Sciences, Onsala Space Observatory
}

Email: carsten.rieck@sp.se

\begin{abstract}
Geodetic VLBI is an independent technique, which does not rely on third parties. This makes it a viable future alternative for time- and frequency transfer over long baselines. Frequency link instabilities in the order of $1.5 \mathrm{e}-15$ for time periods of one day are comparable to those achievable with methods using GNSS carrier-phase observations. Data of the continuous VLBI campaign CONT11 were analyzed and compared to results from GPS PPP analysis on collocated/common clock stations.
\end{abstract}

\section{INTRODUCTION}

The continuous Very Long Baseline Interferometry (VLBI) campaign CONT11 was observed during September 15-29, 2011, involving 13 IVS (International VLBI service for Geodesy and Astrometry) stations. CONT11 is a continuation of the series of very successful continuous VLBI campaigns that were conducted since 1994 . The CONT11 campaign was to acquire state-of-the-art VLBI data in order to demonstrate the highest accuracy of which the current VLBI system is capable of. The collected data set supports high resolution Earth rotation studies, investigations of reference frame stability, investigations of daily to sub-daily site motions, estimation of ultra-rapid dUT1 and the study of time- and frequency transfer techniques [1].

A Previous study of CONT08 campaign data [2] has shown that the frequency transfer capability of geodetic VLBI is comparable to that obtainable using GNSS (Global Navigation Satellite System) carrier phase analysis. During CONT08 both techniques performed frequency link instabilities in the order of $1 \mathrm{e}-15$ for time periods of one day comparing H-maser clocks. Though VLBI is technically complicated and heavy headed, it is an independent technique, which does not rely on third parties. This could make VLBI a viable future alternative for time- and frequency transfer over long baselines. In this paper we present further comparisons using the data set of CONT11.

A requirement for a meaningful comparison of the performance of the two techniques is that VLBI and GNSS

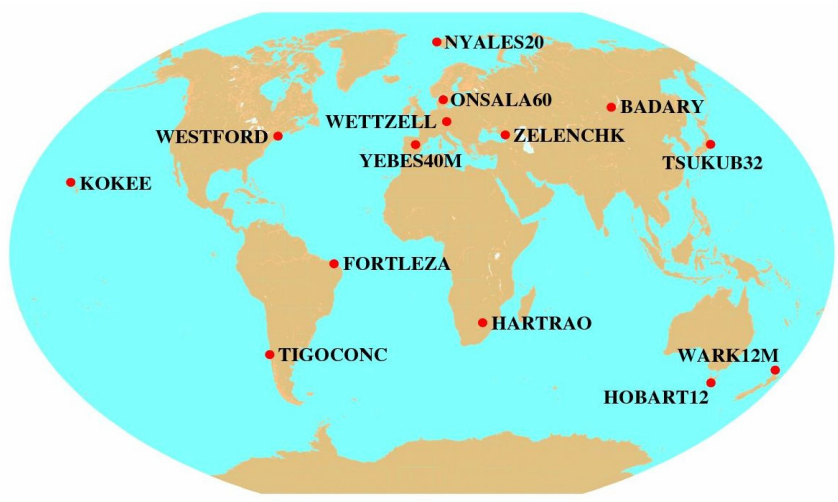

Figure 1. VLBI stations during CONT11. Station WARK12M had to cancel participation due to technical problems. A total of 13 stations successfully collected data. Image taken from [1].

instrumentation is co-located and connected to the same frequency standard, i.e. the same H-maser. The continuous VLBI campaigns provide perfect test beds to compare and evaluate the performance of the two techniques. These campaigns last a few weeks and involve several internationally well distributed co-location stations with the potential to use the same frequency standard for the techniques. During CONT11 eleven out of the thirteen participating stations used the same frequency standard for VLBI and GNSS, with four of the stations also being UTC $(\mathrm{k})$ nodes, providing clock data to the BIPM for contribution to TAI/UTC. Table I presents an overview of the IVS and IGS stations involved during CONT11, Fig. 1 shows the geographical distribution of the stations involved.

\section{METHOD}

The analyzing methods used for CONT11 are similar to those presented in [2] for the CONT08.

VLBI data were analyzed with the VLBI data analysis software Calc/Solve [3]. The setup was a standard network solution using the 15 days of the CONT campaign. Radio source coordinates were fixed to ICRF2, while station 
coordinates were kept fixed on VTRF2008a values. However the station coordinates of Tigo Concepcion and Tsukuba were estimated on a daily basis since these two stations were affected by earthquakes in early 2010 and early 2011, respectively. Earth rotation and orientation parameters were estimated on a daily basis. Atmospheric parameters were estimated as piece-wise linear offsets for zenith wet delays every 20 minutes and horizontal gradients one a day. As constraints we used $50 \mathrm{ps} /$ hour for the zenith wet delays and $0.5 \mathrm{~mm}$ for the gradient offset and $2 \mathrm{~mm} /$ day for the gradient rate. One station clock, Wettzell, was used as reference clock and clock parameters for all other stations were estimated as daily second order polynomials together with additional continuous spline corrections every 20 minutes. The clock constraints were set to $5 \mathrm{e}-14$.

The GNSS data analysis was performed with the NRCANPPP software [4]. GPS data were analyzed for each station using the precise point positioning strategy in a continuous mode for the entire 15 day period, i.e. avoiding 24 hour batches and thus day boundary jumps due to code uncertainties. During the processing final IGS products were used. Zenith wet delays and horizontal gradients were estimated as random walk parameters. Clock parameters were estimated as white noise parameters with $60 \mathrm{~s}$ updates. These clock parameters are relative phase differences to the IGStimescale, which is common to all individual solutions. In order to gain comparable time series with VLBI, all solutions were differenced with the WTZZ solution.

Whereas the PPP clock differences estimates are forced to be continuous over the entire 15 days period ${ }^{1}$, the VLBI derived time series are not and may experience day boundaries. This is due to the fact that correlation and analysis is done in $24 \mathrm{~h}$ batches and that geodetic applications can handle constant clock offsets during the duration of a batch. In order to gain a continuous time series, daily solutions were fitted together with the help of overlapping Solve estimates.

TABLE I. VLBI STATIONS AND IGS COLOCATION DURING CONT11

\begin{tabular}{|c|c|c|c|}
\hline IVS & IGS & $C C^{a}$ & CLOCK \\
\hline HARTRAO & HRAO & YES & EFOS-C 28 \\
\hline KOKEE & KOKB & YES & Sigma Tau \\
\hline NYALES20 & NYAL & YES & APL No 2 \\
\hline ONSALA60 & ONSA & YES & CH1-75A \\
\hline WESTFORD & WES2 & YES & APL No 4 \\
\hline WETTZELL $^{\mathrm{b}}$ & WTZZ/WTZR & YES & EFOS 18 \\
\hline TIGOCONC & CONZ/CONT & YES & EFOS 20 \\
\hline TSUKUB32 & TSKB & YES & Anritsu SA0D05A \\
\hline ZELENCHK & ZELE & YES & VCH-1003A/CH1-80M \\
\hline BADARY & BADG & YES & CH1-80 \\
\hline FORTLEZA & BRFT & $\mathrm{NO}$ & Sigma Tau (VLBI) \\
\hline HOBART12 & HOBA & $\mathrm{NO}$ & VCH-1005A \\
\hline YEBES40M & YEBE & YES & EFOS 66 \\
\hline
\end{tabular}

\footnotetext{
${ }^{1}$ This is only partly true: loss of phase at the receiver due to e.g. loss of signal or receiver reset, forces PPP to estimate a new clock parameter with help of the code phase, which is noisy in nature and introduces uncertainties of about $1 \mathrm{~ns}$ to the clock solution.
}

Link instability analysis uses the fact that the inherent maser-instability is better then most link instabilities for averaging times shorter than approximately one day. Ideally, maser frequency drift is considered to be constant during the duration of the experiment. Thus, individual quadratic clock models were fitted and removed from all time series. The resulting residuals were analyzed using Overlapping Allan Deviation.

The phases of VLBI and PPP clock estimates for each baseline to WETTZELL were also differenced in order to compare the two techniques. A line was estimated, where the trend parameters describes the difference in frequency estimate between the two methods.

\section{RESULTS}

Table II summarizes the results of the study. As for CONT08, also during CONT11 the WETTZELL-ONSALA baseline performs best, both the PPP and the VLBI comparisons reach frequency link instability levels of about $1.2 \mathrm{e}-15$ (overlapping ADEV) for time periods of one day ${ }^{2}$. Both PPP and VLBI derived frequency estimates differ insignificantly from each other, line residuals have a RMS deviation of about 90 ps. Fig. 2 shows the VLBI results and a comparison to the PPP solution for this baseline.
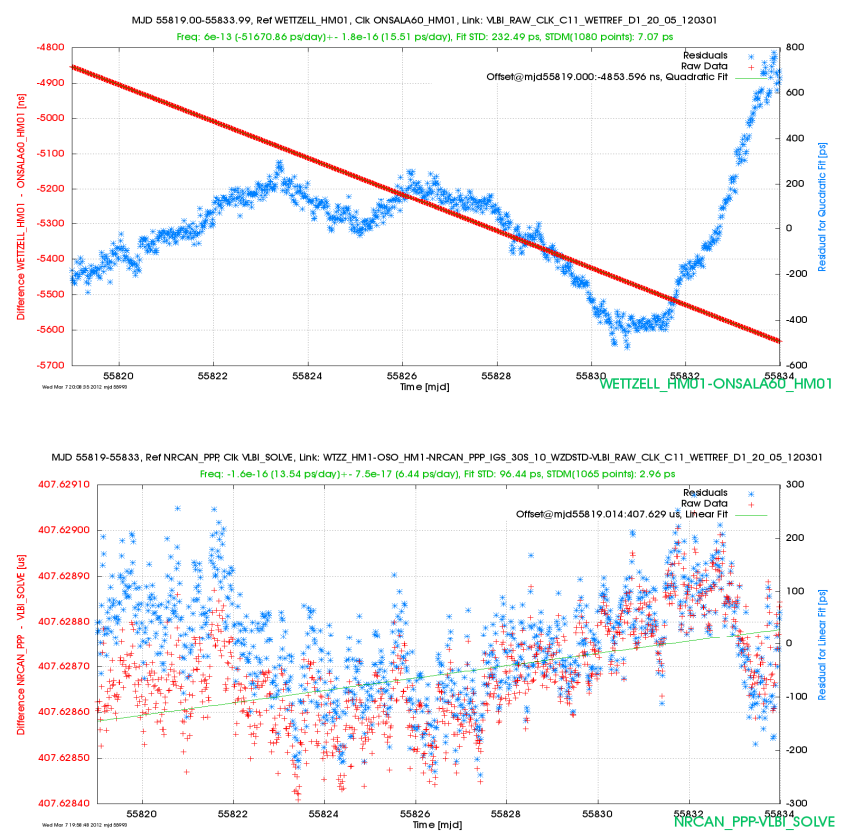

Figure 2. Baseline WETTZELL-ONSALA, MJD 55819-55833. The upper graph shows the VLBI solution, in red the raw phase of the clock difference, blue the residuals to a quadratic model. There is a rapid frequency change MJD55831 due to change in the Onsala clock. The lower graph shows the phase difference between the PPP and the VLBI solutions with a sampling of $1200 \mathrm{~s}$. Both techniques virtually estimate the same relative clock phase with less then 100ps difference in a RMS sense.

\footnotetext{
${ }^{2}$ Best case is based on a reduced data set MJD 55819-55830, Onsala clock has an apparent frequency change on MJD 55831 which violates the assumption of a constant frequency drift of the maser pair. Both, VLBI and PPP estimate the same phase pattern.
} 
TABLE II. CONT11 15 DAYS, MJD 55819-55833, VLBI AND PPP

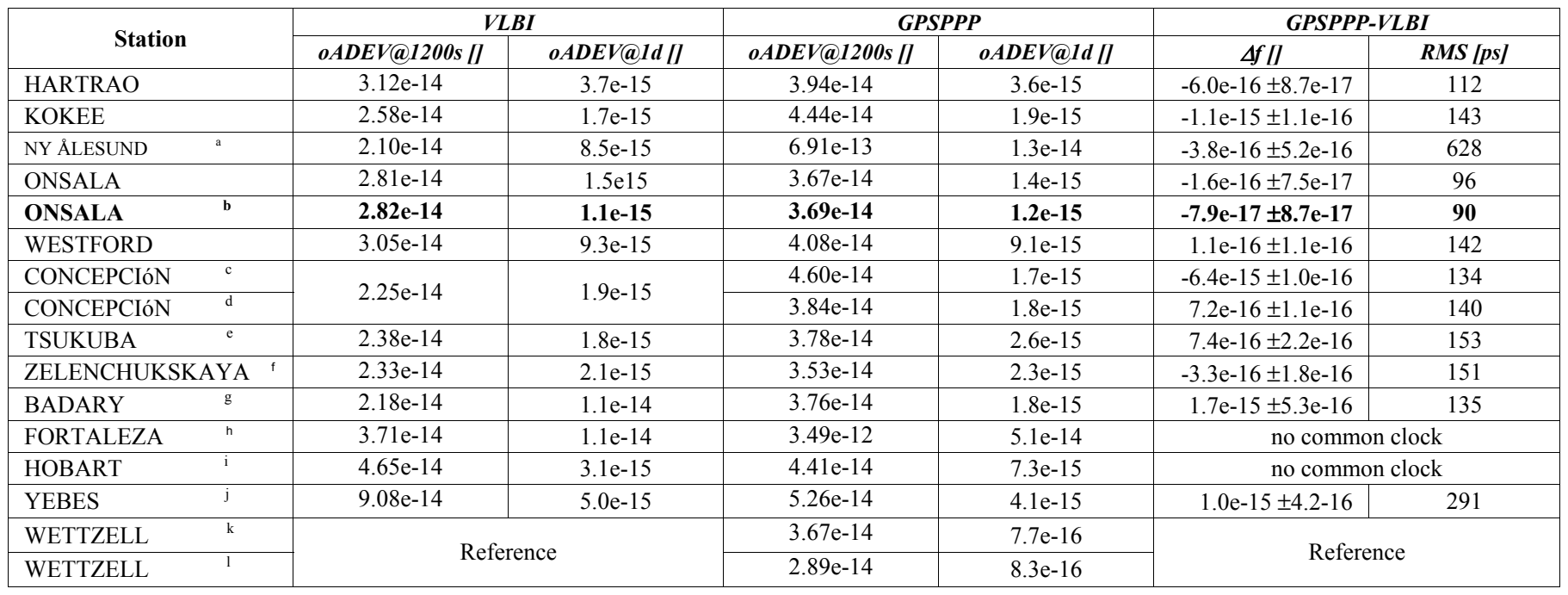

a. PPP CA phase break removed

b. $\quad$ MJD 55819-55830, due to a frequency change of Onsala Maser at MJD 55831 c. IGS CONT

d. IGS CONZ

e. MJD 55826-55826, VLBI clock frequency change at 55825

f. MJ55824-55833, bad VLBI clock prior to 55824

g. MJD 55824-55833, bad VLBI clock prior to 55824, PPP phase break removed

h. no GPS data post to 55825.7, no common clock

i. MJD 55826-55833, VLBI clock frequency change at 55825, no common clock

j. MJD 55819-55826, VLBI clock frequency change at 55827

k. WTZR, short baseline with WTZZ, common clock

l. WTZS, short baseline with WTZZ, common clock

Trend estimates for many of the PPP-VLBI differences in Table II can be considered insignificant. Fig. 3 summarizes the instability measures for the VLBI baselines calculated.

\section{CONCLUSIONS, Future WORK}

VLBI derived relative clock differences for CONT11 have confirmed results from CONT08. Frequency link instabilities are comparable to GPS Carrier Phase processing reaching the 1e-15@1d level. Considering the size and complexity of today's VLBI stations these are excellent figures. It can be anticipated that the next VLBI generation, VLBI2010, will improve VLBI's timing capabilities by providing higher sensitivities, a 24/7 operation and possible real-time capabilties.

The development of tailored analysis methods is an important step to make VLBI acceptable for metrological use. We propose a Kalman-filter based VLBI filter software for continuous clock difference estimation and correct ambiguity resolution. Further, the calibration of VLBI systems would be necessary in order to be useful for time comparisons.

\section{ACKNOWLEDGMENT}

We thank the International VLBI Service for Geodesy and Astrometry and their individuals for organizing and observing CONT11. We thank in particular the VLBI group at the Bundesamt für Kartographie und Geodäsie for their support with the CONT11 data analysis. We acknowledge the National Resources Canada for the NRCAN GPS PPP software.

\section{REFERENCES}

[1] http://ivs.nict.go.jp/mirror/program/cont11/

[2] Rieck et al. "VLBI Time-Transfer using CONT08 Data", EFTF2010

[3] Ma C, Sauber JM, Bell LJ, Clark TA, Gordon D, Himwich WE, Ryan JW (1990) Measurement of horizontal motions in Alaska using Very Long Baseline Interferometry. Journal of Geophysical Research, 95(B13), 21991-22011.

[4] Orgiazzi D, Tavella P, Lahaye F (2005) Experimental Assessment of the Time Transfer Capability of Precise Point Positioning (PPP). In: Proc. of the Joint IEEE Intl. FCS and the PTTI, 337-345.

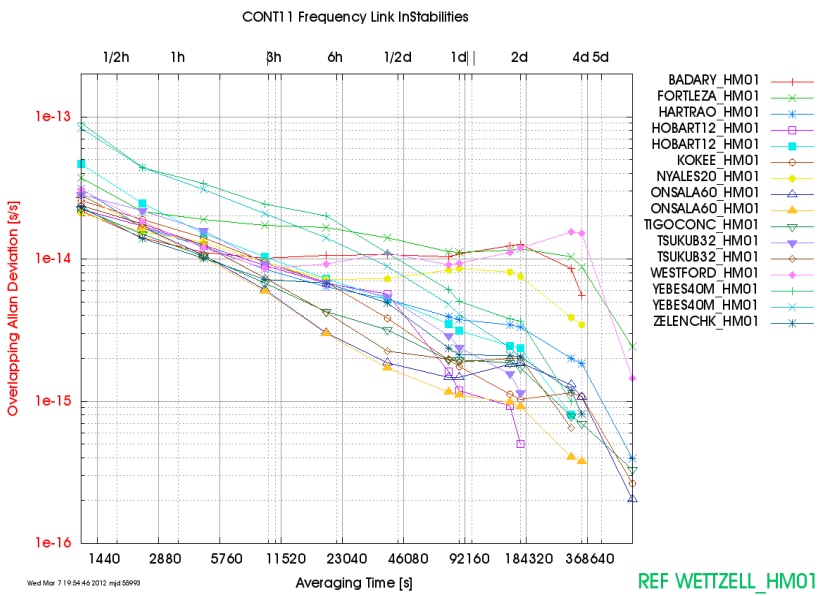

Figure 3. CONT11 Frequency link instabilities, overlapping ADEV. For some stations more results are shown than those reported in Table II. Hobart 1:(MJD 55819-55824), 2:(MJD 55826-55833),

Tsukuba 1:(MJD 55819-55824), 2:(MJD 55826-55833),

Yebes 1:(MJD 55819-55826), 2:(MJD 55828-55833),

Onsala 1:(MJD 55819-55833), 2:(MJD 55819-55830). 